\title{
The Importance of Ice Vertical Resolution for Snowball Climate and Deglaciation
}

\author{
DORIAN S. ABBOT \\ Department of Geophysical Sciences, University of Chicago, Chicago, Illinois \\ IAN EISENMAN \\ Division of Geological and Planetary Sciences, California Institute of Technology, and Department of \\ Atmospheric Sciences, University of Washington, Seattle, Washington \\ RAYMOND T. PIERREHUMBERT \\ Department of Geophysical Sciences, University of Chicago, Chicago, Illinois
}

(Manuscript received 24 February 2010, in final form 28 June 2010)

\begin{abstract}
Sea ice schemes with a few vertical levels are typically used to simulate the thermodynamic evolution of sea ice in global climate models. Here it is shown that these schemes overestimate the magnitude of the diurnal surface temperature cycle by a factor of $2-3$ when they are used to simulate tropical ice in a Snowball earth event. This could strongly influence our understanding of Snowball termination, which occurs in global climate models when the midday surface temperature in the tropics reaches the melting point. A hierarchy of models is used to show that accurate simulation of surface temperature variation on a given time scale requires that a sea ice model resolve the $e$-folding depth to which a periodic signal on that time scale penetrates. This is used to suggest modifications to the sea ice schemes used in global climate models that would allow more accurate simulation of Snowball deglaciation.
\end{abstract}

\section{Introduction}

At least twice during the Neoproterozoic, about 710 million years ago and about 635 million years ago, the climate was so cold that there were glaciers at sea level in the tropics (Evans 2000; Trindade and Macouin 2007; Hoffman and Li 2009). According to the Snowball earth hypothesis, these glaciations were global and contemporaneous, the oceans were entirely covered with ice for millions of years, and the climate recovered only when volcanic outgassing slowly brought the partial pressure of carbon dioxide $\left(p \mathrm{CO}_{2}\right)$ to such extremely high values that ice melt could occur in the tropics, leading to global deglaciation (Kirschvink 1992; Hoffman et al. 1998). A crucial outstanding aspect of the Snowball earth story is a full explanation of deglaciation, including, for example, the quantity of $\mathrm{CO}_{2}$ required for deglaciation

Corresponding author address: Dorian Abbot, Department of Geophysical Sciences, University of Chicago, 5734 South Ellis Ave., Chicago, IL 60637.

E-mail: abbot@uchicago.edu
(Pierrehumbert 2004) and whether other factors such as surface dust (Abbot and Pierrehumbert 2010) and dust aerosol (Abbot and Halevy 2010) are required to cause deglaciation at a $p \mathrm{CO}_{2}$ consistent with observational evidence.

Although atmospheric models of varying complexity, ranging from radiative-convective models (Abbot and Halevy 2010) to energy balance models (Caldeira and Kasting 1992; Hyde et al. 2000; Pollard and Kasting 2005; Lewis et al. 2006) to global climate models (GCliMs) (Pierrehumbert 2004, 2005; Le Hir et al. 2007; Abbot and Pierrehumbert 2010), have been used to study Snowball deglaciation, the tests used to characterize the state of the climate that would lead to deglaciation have mostly been relatively simple and have not always been consistent with one another. For example, while annual-mean tropical surface temperatures exceeding $0^{\circ} \mathrm{C}$ are manifestly a sufficient condition for Snowball deglaciation, Caldeira and Kasting (1992) consider an annual-mean tropical surface temperature of $-10^{\circ} \mathrm{C}$ a sufficient condition for Snowball deglaciation, whereas Pierrehumbert (2005) argue that an annual-mean tropical surface temperature 
of $0^{\circ} \mathrm{C}$ is more appropriate, and that at the very least summertime mean surface temperatures of $0^{\circ} \mathrm{C}$ in the subtropics are necessary for deglaciation. Other authors, using sea ice parameterizations with various differing assumptions, use either the initiation of sea ice melt (Le Hir et al. 2007) or the total melting of all sea ice (Hyde et al. 2000; Pollard and Kasting 2005; Abbot and Pierrehumbert 2010) as a condition for deglaciation. Although these tests might seem similar, in fact they can result in vastly different estimates of the atmospheric and surface conditions that would cause deglaciation.

Because of the logarithmic dependence of radiative forcing on $p \mathrm{CO}_{2}$, small differences in the surface conditions required to cause Snowball deglaciation can have an enormous effect on the $p \mathrm{CO}_{2}$ required to cause deglaciation. For example if the climate sensitivity in a Snowball near deglaciation were as high as the climate sensitivity today, a change in the surface conditions required for deglaciation of only a few degrees Celsius would change the $p \mathrm{CO}_{2}$ required for deglaciation by a factor of 2 . This is crucially important for the comparison of the $p \mathrm{CO}_{2}$ required to cause deglaciation in models with the $p \mathrm{CO}_{2}$ implied by $\Delta^{17} \mathrm{O}$ values preserved in Neoproterozoic barites and evaporites (e.g., Bao et al. 2008, 2009). Additionally, as long as the sinks of $\mathrm{CO}_{2}$ during a Snowball are small and the $p \mathrm{CO}_{2}$ required for deglaciation is only on the order of 0.1 bar, the Snowball lifetime should scale linearly with the $p \mathrm{CO}_{2}$ required for deglaciation, which is essential for comparison with geochronological data (Hoffman and Li 2009). If these two conditions are not met, errors in model estimates of the $p \mathrm{CO}_{2}$ required for deglaciation would cause even larger errors in the associated Snowball lifetime estimates (Higgins and Schrag 2003; Le Hir et al. 2008).

Since the surface ice conditions that would lead to deglaciation are a pivotal aspect of the Snowball story, but have not been satisfactorily studied, we will consider them in this paper. As an initial effort, we will focus here largely on the simulation of the surface temperature diurnal cycle by the ice parameterizations used in GCMs. Because the surface temperature exceeding the melting point at noon is a necessary condition for deglaciation, the proper simulation of the ice surface diurnal cycle is crucially important for determining deglaciation conditions correctly.

Noon surface temperatures exceeding the melting point are not, in general, a sufficient condition for deglaciation, since the meltwater that they would produce could refreeze at night. The ice parameterizations in GCMs, however, generally assume that surface meltwater is immediately evacuated so that a "melt-ratchet effect," with daytime melting and no nighttime freezing, can act to decrease ice thickness.
Sea ice schemes in GCMs solve the Stefan problem of sea ice growth and melting (Stefan 1891). These schemes are generally based on a simplification of Maykut and Untersteiner (1971) described by Semtner (1976) that attempts to allow reasonable simulation of the seasonal cycle in ice surface temperature and ice thickness in the modern climate with only a few vertical levels (typically between zero and four prognostic temperatures computed to approximate the vertical profile). In the modern climate sea ice is typically only a few meters thick, but tropical sea ice during a Snowball earth event would likely have been hundreds of meters thick, and it would likely have consisted of glacier ice (compacted snow) as well as frozen seawater (Goodman and Pierrehumbert 2003; Goodman 2006). It is possible that tropical sea ice during a Snowball event could have been as thin as a few meters (Pollard and Kasting 2005), although this solution may exist only in a very narrow range of ice albedo and solar penetration parameters (Warren and Brandt 2006; Pollard and Kasting 2006). Although it may seem obvious that a scheme designed for thin ice and using only a few vertical levels might not produce reasonable results when used to simulate thick ice, this issue has not been considered previously. We will argue below that the vertical resolution of the sea ice scheme is of grave importance for simulating the surface temperature diurnal cycle and ultimately for determining the conditions that would lead to Snowball deglaciation.

The plan of the manuscript is as follows. In section 2 we show that deglaciation of the Community Atmosphere Model (CAM) GCM occurs when the mean tropical surface temperature is less than $-10^{\circ} \mathrm{C}$ as a result of a very large surface temperature diurnal cycle. We then use the single-column version of CAM (hereafter SCAM) to show that the surface temperature diurnal cycle is drastically reduced when the sea ice scheme vertical resolution is increased (section 3), which may imply that deglaciation in CAM occurs at unrealistically low mean surface temperatures. In section 4 we use an idealized sea ice model to fully understand these results and determine the criteria that should be used when setting the sea ice resolution. In section 5 we discuss our results and in section 6 we summarize our main conclusions.

\section{Large snowball diurnal cycle in global climate models}

Recent simulations of Snowball climate in the Fast Ocean Atmosphere Model (FOAM), version 1.5, and the National Center for Atmospheric Research's (NCAR's) Community Atmosphere Model, version 3.1, have shown that complete loss of sea ice is initiated in both of these models when the $p \mathrm{CO}_{2}$ is increased enough or the surface 
(a) Surface Air Temperature Over Ice $\left[{ }^{\circ} \mathrm{C}\right]$

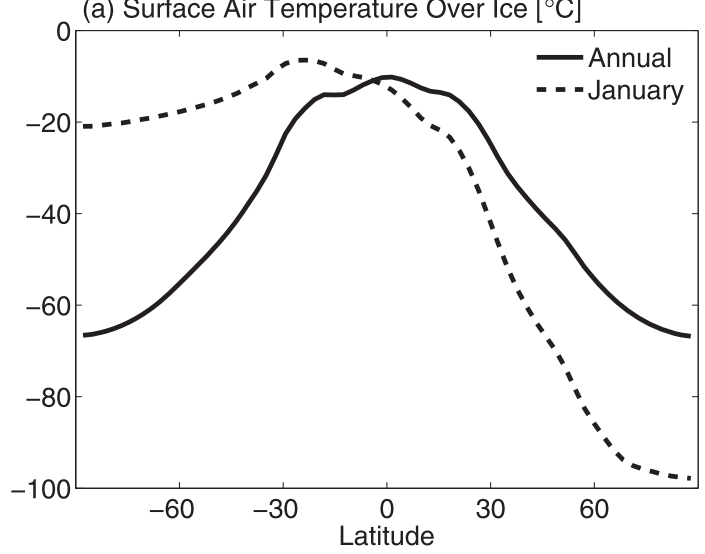

(b) Ice Surface Melt Rate $\left[\mathrm{cm} \mathrm{day}^{-1}\right]$

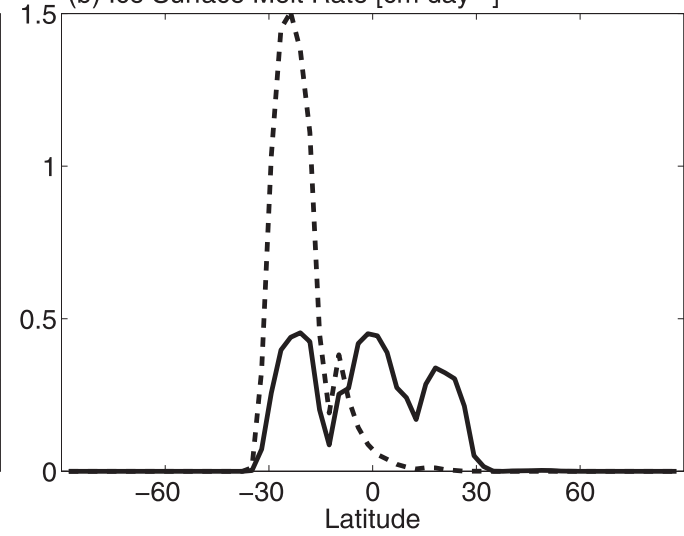

FIG. 1. Zonal average of annual-mean (solid) and January-mean (dashed) (a) surface air temperature over ice and (b) ice surface melt rate for the first $5 \mathrm{yr}$ of a simulation of Snowball climate in the CAM GCM with sea ice as a lower boundary condition and $p \mathrm{CO}_{2}=0.1$ bar.

is sufficiently darkened by dust that the annual-mean tropical surface temperature reaches $\sim-15$ to $\sim-10^{\circ} \mathrm{C}$ (Abbot and Pierrehumbert 2010). For example, complete loss of sea ice occurred in a CAM simulation with $p \mathrm{CO}_{2}=0.1$ bar and no dust surface darkening effects. Large positive cloud feedbacks allowed CAM to deglaciate at a lower $p \mathrm{CO}_{2}$ than other GCMs typically do (Abbot and Pierrehumbert 2010). Figure 1 shows the zonal average of the annual-mean and January-mean surface air temperature over ice and the ice surface melt rate averaged over the first five years of this CAM simulation. The maximum annual-mean surface air temperature is $-10.2^{\circ} \mathrm{C}$ and occurs at the equator and the maximum monthly-mean surface air temperature is $-6.5^{\circ} \mathrm{C}$ and occurs in January in the southern subtropics (Fig. 1a). A large annual cycle in subtropical melt rate produces an annual-mean subtropical melt rate that is roughly equal to the annual-mean equatorial melt rate (Fig. 1b). Since the sea ice is initially thinnest near the equator in the model, sea ice first completely melts there, exposing the underlying ocean, and then the ice coverage recedes poleward.

To understand why melting of sea ice and deglaciation occurs at such a low mean surface temperature, we show 10 days of the 2-hourly surface air temperature time series from a typical grid point at the equator that is far from any continent in this CAM simulation (Fig. 2a). The model produces a surface temperature diurnal cycle with a characteristic magnitude of $15^{\circ}-20^{\circ} \mathrm{C}$ that is large enough so that the surface temperature reaches the melting point and surface melting occurs at midday (Fig. 2b). The model assumes that the water from melted ice is immediately evacuated and does not refreeze during the cold night, which leads to a progressive loss of sea ice and eventual deglaciation. At lower $p \mathrm{CO}_{2}$ the model produces even larger diurnal cycles, but since the mean temperature is lower, melting does not occur. For example, for $p \mathrm{CO}_{2}=$ $100 \mathrm{ppm}$ the magnitude of the diurnal cycle of tropical surface temperature is typically $25^{\circ}-30^{\circ} \mathrm{C}$ (not shown).

In addition to sea ice, CAM also contains a glacial ice scheme. Glacial ice is the land surface boundary condition used in Antarctica and Greenland when CAM is run in a modern configuration, whereas sea ice is modeled using a thermodynamic scheme described by Bitz and Lipscomb (1999). Sea ice can grow and melt but glacial ice cannot. The two ice types have similar thermal conductivities but vastly different vertical resolutions: the sea ice in the simulations described in this section is about $10-15 \mathrm{~m}$ thick in the tropics and the

(a) Surface Temperature $\left[{ }^{\circ} \mathrm{C}\right]$
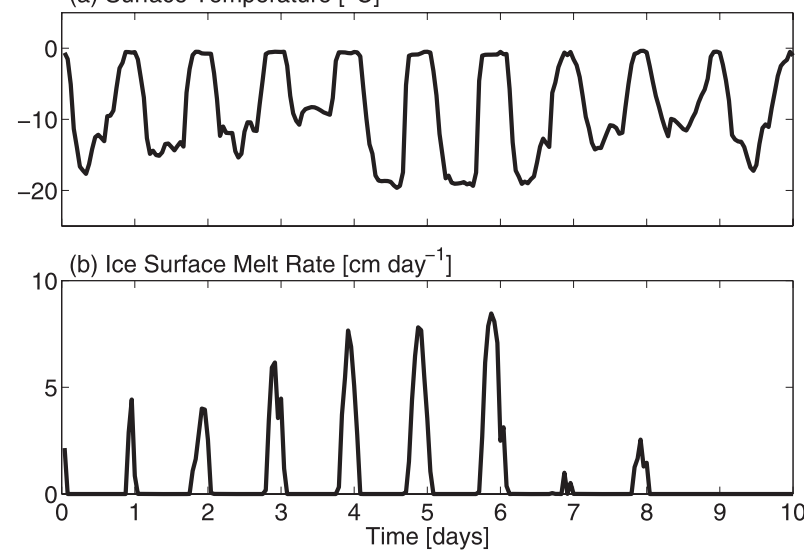

FIG. 2. Time series of (a) surface air temperature and (b) ice surface melt rate for 10 typical days at a grid point at the equator that is far from any continent in a simulation of Snowball climate in the CAM GCM with sea ice as a lower boundary condition and $p \mathrm{CO}_{2}=0.1$ bar. 
sea ice scheme has four evenly spaced layers, while the glacial ice has 10 unevenly spaced layers (concentrated at the top) within the top $0.8 \mathrm{~m}$ of the ice. Interestingly, the glacial ice scheme produces a surface temperature diurnal cycle with a typical magnitude that is one-third to one-half of the diurnal cycle produced by CAM's sea ice scheme when the model is run in exactly the same configuration except with glacial ice replacing sea ice (not shown). Given that the thermal heat capacities of sea ice and glacial ice are roughly the same, it is surprising that there is such a large difference in the diurnal cycles the model produces in the two cases. In section 3 we will show that the difference in diurnal cycle simulation between the two ice schemes is mainly due to the drastically higher vertical resolution near the surface of the glacial ice model as compared to the sea ice model.

\section{Effect of increasing sea ice vertical resolution}

In this section we show that the number of sea ice layers has a profound effect on the diurnal cycle when a single grid point of CAM's sea ice scheme is coupled to SCAM (Hack et al. 2004). SCAM contains all the cloud, convection, and radiation representations of CAM, but dynamical terms must be supplied as boundary conditions. We choose model parameters to correspond with typical tropical conditions during a hypothetical Snowball earth event. We drive the model with diurnally and seasonally varying shortwave radiation for modern orbital parameters at the equator multiplied by 0.94 to represent the reduced solar energy output during the Neoproterozoic and we set $p \mathrm{CO}_{2}$ to $100 \mathrm{ppm}\left(10^{-4}\right.$ bar $)$. We specify a dry atmospheric heat transport of -50 $\mathrm{W} \mathrm{m}{ }^{-2}$ and a moist atmospheric heat transport of $-10 \mathrm{~W} \mathrm{~m}^{-2}$ to the atmospheric column using the method of Abbot and Tziperman (2008, 2009). We disable the model's sea ice solar penetration scheme because large changes in the simulation of mean surface temperature occur when the vertical resolution is changed with this scheme active. Although this affects the mean surface temperature the model simulates, it does not significantly affect the magnitude of the surface temperature diurnal cycle.

We initialize the model with a sea ice thickness of $10 \mathrm{~m}$. Based on results we will discuss in section 4, we expect that 60 evenly spaced vertical levels should be required to resolve the penetration depth of the diurnal cycle. Increasing the model's vertical resolution from its standard value of four levels to 60 levels reduces the diurnal cycle by a factor of about 2-3 (Fig. 3), which is consistent with the reduction in the diurnal cycle of glacial ice relative to sea ice (section 2). One way to

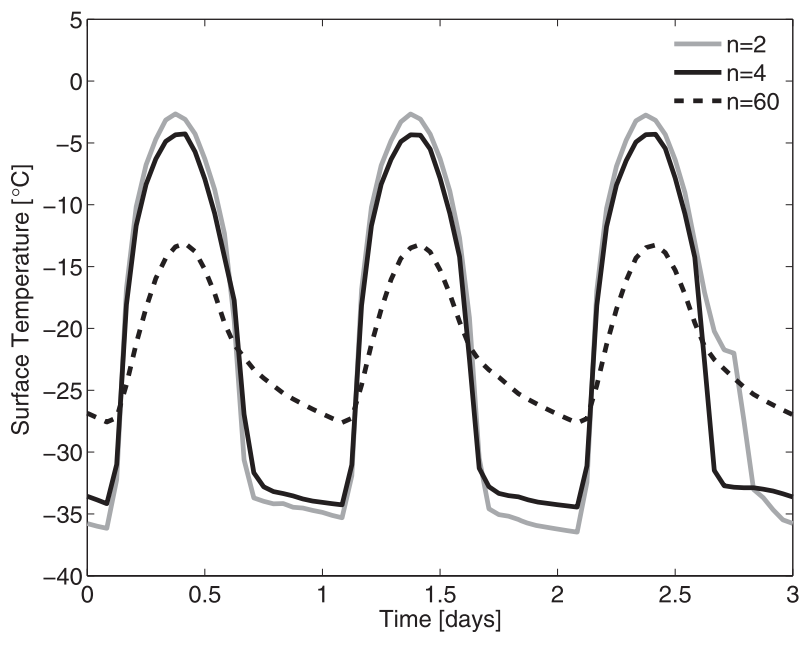

FIG. 3. Surface temperature diurnal cycle in NCAR's thermodynamic sea ice model coupled to the single-column version of CAM (SCAM) for different numbers of sea ice levels $(n)$. The model is driven with realistic diurnally varying Neoproterozoic tropical solar radiation.

conceptualize the decrease in the diurnal temperature cycle as the vertical resolution is increased is that increasing the vertical resolution increases the effective heat capacity of the ice (section 4). This explains why the surface temperature decreases much more slowly during night at high vertical resolution and why the phase lag between the solar forcing and the temperature response increases as the resolution is increased (Fig. 3).

\section{Importance of diffusive penetration depth}

In this section we will consider a single-column thermodynamic sea ice model that follows Semtner (1976), which is similar to the thermodynamic component of the sea ice schemes in most GCMs, with three small changes. First, we do not include snow because there should be a net ablation zone in the tropics of a Snowball event in which ice would experience only transient snow cover that would quickly sublimate (Abbot and Pierrehumbert 2010). Second, we do not allow penetration of solar radiation because the solar absorption parameterization of Semtner (1976) is specifically designed for low vertical resolution and is not appropriate when the resolution approaches the extinction depth. Third, we do not include the thermal effect of brine pockets.

Following Semtner (1976), we solve the diffusion equation within the ice with a constant number of equally spaced vertical levels that expand or shrink as the ice grows and melts. Surface heat balance is enforced at the top of the ice by solving the following equation for $T_{s}$, the surface temperature: 


$$
F(t)-F_{1 \mathrm{w}}\left(T_{s}\right)+\frac{C_{i} k\left(T_{1}-T_{s}\right)}{h /(2 n)}=0 .
$$

Here $C_{i}$ is the volumetric heat capacity of ice, $\kappa$ is the thermal diffusivity of ice, $T_{1}$ is the temperature of the uppermost ice layer, $h$ is the ice thickness, and $n$ is the number of layers. The idealized forcing function, $F(t)$, in Eq. (1) represents a combination the shortwave (SW), latent heat $(\mathrm{LH})$, and sensible heat $(\mathrm{SH})$ fluxes:

$$
F(t)=(1-\alpha) \mathrm{SW}-\mathrm{LH}-\mathrm{SH},
$$

where $\alpha$ is the surface albedo. To explore the behavior of the scheme as the vertical resolution is increased, we choose the following idealized form of the diurnal cycle in $F(t)$ :

$$
F(t)=F_{1}+F_{2} \cos [2 \pi(d-0.5)]
$$

where $F_{1}$ and $F_{2}$ are constants and $d$ is the time in days. At noon the net heat flux into the ice from above reaches a maximum of $F_{1}+F_{2}$ and at midnight it reaches a minimum of $F_{1}-F_{2}$. We specify the net longwave heat flux at the ice upper boundary $\left[F_{\mathrm{lw}}\left(T_{s}\right)\right]$ in Eq. (1) in the following way:

$$
F_{\mathrm{lw}}\left(T_{s}\right)=(1-\varepsilon) \sigma T_{S}^{4} .
$$

We assume that a fraction $(\varepsilon)$ of the upward longwave radiation is absorbed by the atmosphere, reemitted back downward, and absorbed by the surface. Finally, we also set the temperature at the bottom of the ice to the freezing point of seawater $\left(-1.8^{\circ} \mathrm{C}\right)$ and the heat flux into the bottom of the ice to 0 for simplicity. When Eq. (1) gives a surface temperature $T_{S}>0^{\circ} \mathrm{C}$, we assume surface ablation occurs and set the surface temperature to $T_{S}=0^{\circ} \mathrm{C}$.

As an illustrative example, we choose the parameters $F_{1}=80 \mathrm{~W} \mathrm{~m}^{-2}, F_{2}=40 \mathrm{~W} \mathrm{~m}^{-2}$, and $\varepsilon=0.7$. The qualitative behavior of the system is not sensitive to these choices. We use an initial ice thickness of $10 \mathrm{~m}$, which allows us to investigate the model at high vertical resolution without incurring prohibitive numerical cost. The tropical ice thickness during a Snowball event is a matter of debate (e.g., Goodman and Pierrehumbert 2003; Pollard and Kasting 2005) but may have been as much as $O(100-1000 \mathrm{~m})$. Increasing the ice initial thickness above $10 \mathrm{~m}$ leads to small decreases in the surface temperature since the average heat flux from the ice to the surface scales like $\mathrm{h}^{-1}$, but it does not significantly affect the surface temperature diurnal cycle.

The near-surface and surface temperatures of the model are extremely sensitive to vertical resolution. For example, the magnitude of the surface temperature diurnal (a) Temperature $\left[{ }^{\circ} \mathrm{C}\right]$

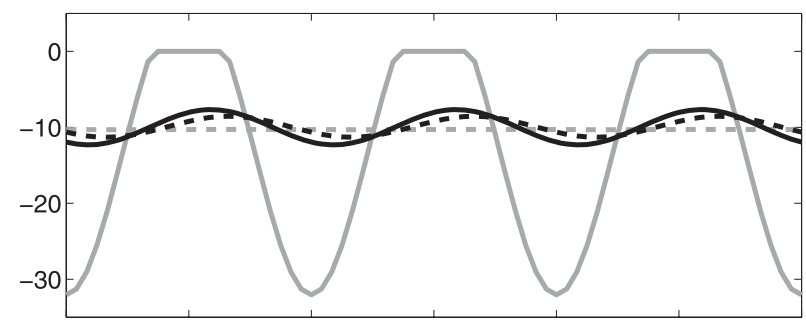

(b) Surface Melt Rate $\left[\mathrm{mm}\right.$ day $\left.^{-1}\right]$

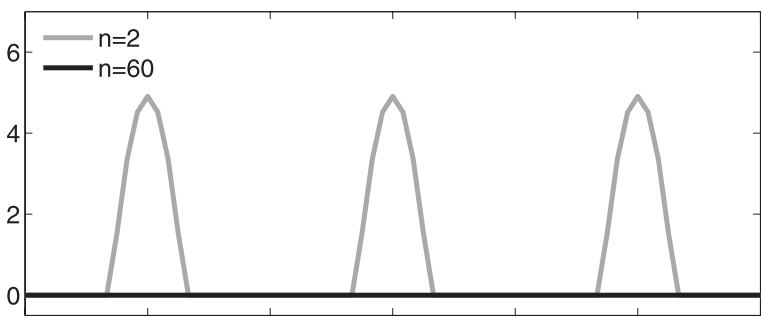

(c) Heat Flux from Ice to Surface $\left[\mathrm{W} \mathrm{m}^{-2}\right]$

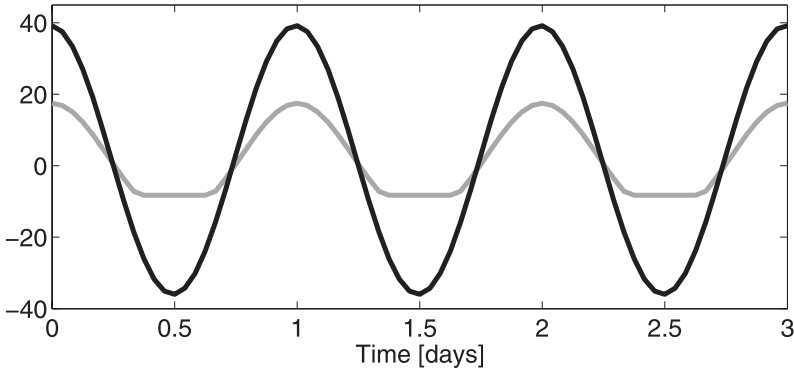

FIG. 4. Diurnal cycle of (a) the surface temperature (solid) and the temperature of the uppermost ice layer (dashed), (b) the surface melt rate, and (c) the diffusive heat flux from the ice interior to the surface when the resolution of the Semtner sea ice model (section 4 ) is $5 \mathrm{~m}$ (gray; $n=2$ ) and when the model resolution is $0.17 \mathrm{~m}$ (black; $n=60$ ). The diurnal forcing is described by Eqs. (1) and (3).

cycle is about $5^{\circ} \mathrm{C}$ when 60 vertical levels are used but is more than $30^{\circ} \mathrm{C}$ when only two vertical levels are used (Fig. 4a). As the number of vertical levels is increased, the magnitude of the surface temperature diurnal cycle steadily decreases until it saturates when roughly 60 vertical levels are used (Fig. 5). Even though vertical resolution strongly affects the diurnal cycle, the mean surface temperature is roughly $-10^{\circ} \mathrm{C}$ at all resolutions.

The ice resolution affects the surface temperature through the diffusive heat flux from the uppermost ice level to the surface in Eq. (1) (the third term on the lefthand side). As the ice resolution increases (grid spacing decreases), the heat flux from the ice to the surface increases at night and decreases (becomes more negative) during the day (Fig. 4c), which significantly moderates the diurnal cycle. This happens because although the difference between the surface temperature and the uppermost 


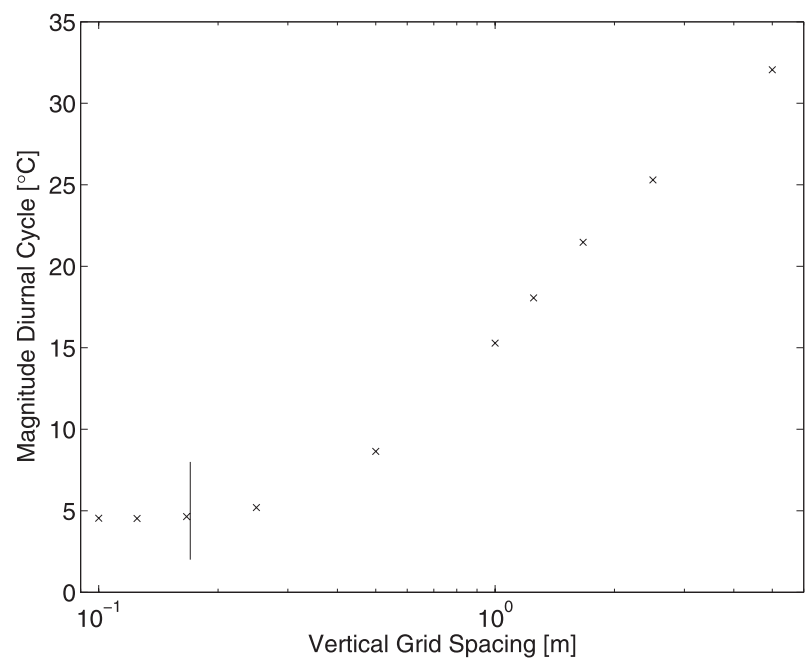

FIG. 5. Magnitude of the diurnal cycle (maximum - minimum) produced by the Semtner sea ice model (section 4) as a function of model vertical resolution. The ice thickness is $10 \mathrm{~m}$, so a vertical grid spacing of $0.1 \mathrm{~m}$ corresponds to 100 vertical levels and a vertical grid spacing of $5 \mathrm{~m}$ corresponds to two vertical levels. The vertical line on the plot denotes the $e$-folding depth to which a periodic diurnal signal should penetrate [Eq. (5)].

ice temperature decreases as the resolution increases (Fig. 4a), it does not do so as fast as the ice layers thin $\left(\propto n^{-1}\right)$. In this way, although the individual heat capacity of the uppermost layer decreases as the resolution increases, the effective heat capacity of the ice as a whole increases as the ice resolution increases.

Since the surface temperature reaches the melting point at noon at low vertical resolution (large grid spacing) but not at high vertical resolution (small grid spacing) (Fig. 4a), the magnitude of the diurnal cycle has a huge effect on the surface melt rate (Figs. $4 b$ and 6). This results in steady ice loss and eventual deglaciation at low vertical resolution, but not at high vertical resolution (Fig. 7). Experimentation with a broad range of other parameter choices shows that this behavior is not sensitive to the particular parameter choice we have made here (not shown). This simple example shows how the coarse-resolution sea ice parameterizations employed in GCMs can lead to erroneous Snowball earth deglaciations.

The enormous effect of sea ice vertical resolution on the diurnal temperature cycle can be understood by considering the diffusive penetration depth of a periodic signal. When a diffusive medium is periodically forced from a boundary, the periodic signal penetrates into the medium to an $e$-folding depth, $z^{*}$ described by

$$
z^{*}=\sqrt{\frac{\kappa P}{\pi}}
$$

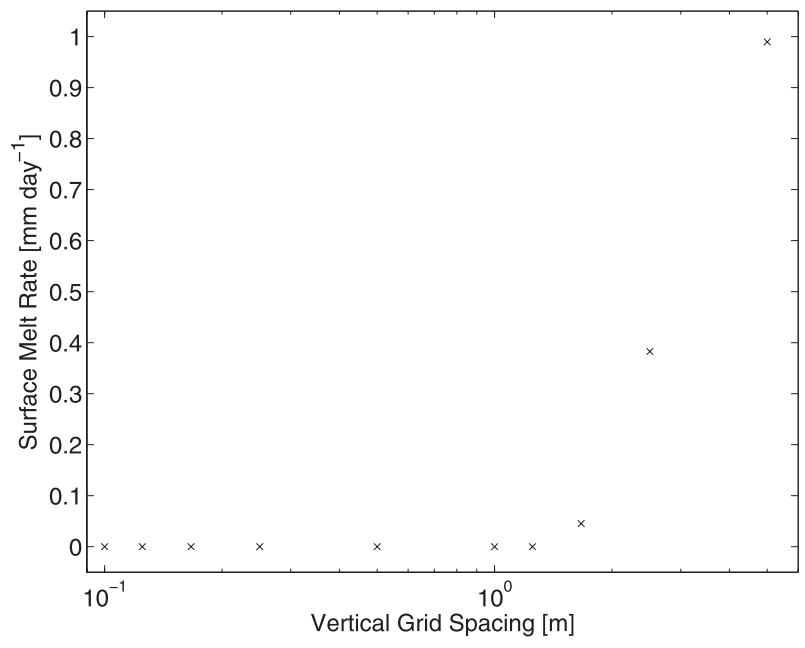

FIG. 6. Average surface melt rate when the Semtner sea ice model is forced by a diurnal cycle as a function of model vertical resolution. A nonzero surface melt rate, which would lead to Snowball deglaciation, occurs at low vertical resolution but not at high vertical resolution.

where $\kappa$ is the thermal diffusivity and $P$ is the period of the forcing signal (see the appendix for details). For ice, $\kappa \approx 10^{-6} \mathrm{~m}^{2} \mathrm{~s}^{-1}$, so $z^{*} \approx 17 \mathrm{~cm}$ for diurnal forcing. This length scale is plotted as a vertical line in Fig. 5. Clearly the model must resolve the $e$-folding depth of penetration of the diurnal cycle in order to produce an accurate simulation of the diurnal surface temperature cycle.

\section{Discussion}

Our results indicate that the sea ice schemes used in GCMs may hugely overestimate the surface temperature diurnal cycle in a Snowball climate. Given the importance

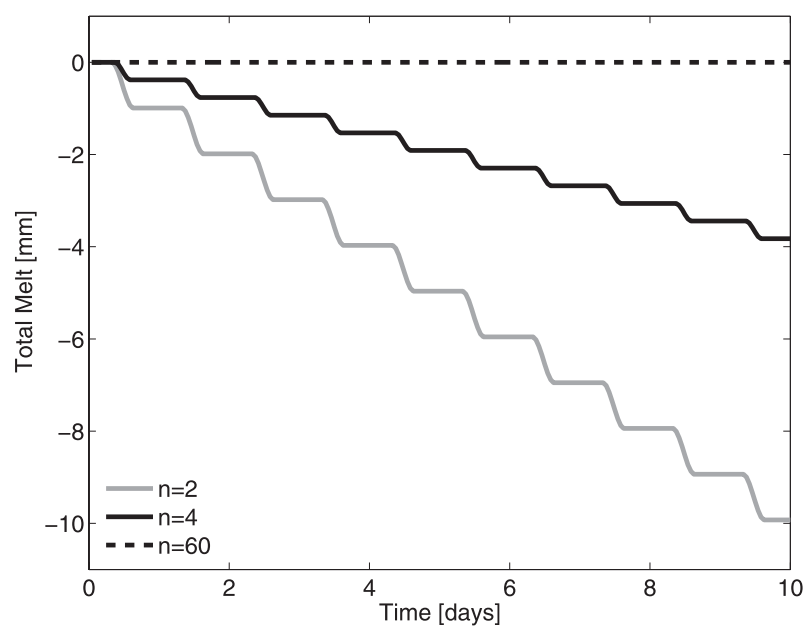

FIG. 7. Time series of total surface melt produced by the Semtner sea ice model for different numbers of vertical levels. 
of noontime temperature for Snowball deglaciation, this means that previous GCM deglaciation results (Pierrehumbert 2004, 2005; Le Hir et al. 2007; Abbot and Pierrehumbert 2010) should be revisited. This point deserves emphasis because previous GCM simulations have required at least a few tenths of a bar of $\mathrm{CO}_{2}$ to deglaciate whereas $\Delta^{17} \mathrm{O}$ values preserved in barites and evaporites suggest that $p \mathrm{CO}_{2}$ was only a few hundredths of bar during and just after the Snowball event $\sim 635$ million years ago (Bao et al. 2008, 2009). Therefore, the difficulty of producing a Snowball deglaciation in a $\mathrm{GCM}$ at a $\mathrm{CO}_{2}$ concentration consistent with the observations of Bao et al. $(2008,2009)$ may be even more acute than previously thought, making the potential of surface (Abbot and Pierrehumbert 2010) and aerosolized (Abbot and Halevy 2010) dust to reduce the global albedo particularly important. If GCMs are to be used to simulate Snowball deglaciation in the future, then their sea ice schemes should be altered to increase the number of levels near the top of the sea ice so that the penetration depth of the diurnal cycle is resolved. The temperature profile in our simulations is very close to linear below one to two penetration depths $(\sim 0.5 \mathrm{~m})$, so that very few levels would be needed below $\sim 0.5 \mathrm{~m}$.

It is important to note that accurate simulation of the surface temperature diurnal cycle does not ensure accurate simulation of all deglaciation processes. In particular, the assumption that meltwater is instantly removed from the ice surface, common to most GCM sea ice schemes, may be problematic (see further discussion below). While this assumption might be acceptable in certain situations for thin ice with surface meltwater that slowly forms over months during the melting season, it is far less likely to be realistic for surface meltwater on thick ice that is generated over a few hours at midday and then subjected to subfreezing temperatures only hours later. This is an illuminating example of the fact that GCMs do not always yield more accurate results than simpler models, particularly when used to simulate climates vastly different from the modern one, since the many assumptions made in GCM schemes can become unreasonable.

Our results may be important in climatic situations other than Snowball termination. For example, they indicate that Snowball initiation in GCMs (Poulsen et al. 2001; Poulsen 2003; Donnadieu et al. 2004; Poulsen and Jacob 2004; Romanova et al. 2006; Voigt and Marotzke 2010) and the possibility of a stable climate with open ocean in the tropics surrounded by ice (Hyde et al. 2000) should be reevaluated using sea ice schemes that resolve the penetration depth of the diurnal cycle. Additionally, it is possible that coarse sea ice vertical resolution could affect the simulation of climate over sea ice and sea ice extent predictions in modern and future climate simulations through the rectification of errors in the simulation of the diurnal cycle. The number of vertical levels required to resolve the annual cycle has been investigated in previous studies (e.g., Semtner 1976; Bitz et al. 2001), but the influence of vertical resolution on the simulation of the diurnal cycle, which is also included in the forcing in most GCMs, has received less attention. More work, however, would be needed to determine the veracity of this idea.

Here we have investigated the effect of vertical resolution on the diurnal cycle of bare sea ice. We did this because there should be a net ablation zone in the tropics of a Snowball, leading to bare sea ice in this region, and the tropics is the region where Snowball deglaciation is most likely to initiate (Abbot and Pierrehumbert 2010). If we were to extend this work to the other climatic contexts mentioned above, we would have to consider snow as well. The full investigation of the effects of snow is beyond the scope of the current work, but we feel it is important to discuss a few issues here. First, even if the snow rate is fairly low $\left(\sim 0.5 \mathrm{~mm} \mathrm{day}^{-1}\right)$, enough snow can accumulate to insulate sea ice and prevent the type of noon melting described here. Since many current models do not use any internal layers for snow, however, the snow itself could experience exaggerated diurnal cycles and exaggerated midday melting. For example, if we assume a thermal diffusivity of snow of $4.5 \times 10^{-7} \mathrm{~m}^{2} \mathrm{~s}^{-1}$ (Maykut and Untersteiner 1971), then the $e$-folding depth to which a diurnal signal will penetrate for snow is $11 \mathrm{~cm}$. Therefore, a model without internal snow layers will overestimate the diurnal cycle if snow accumulates to a thickness of greater than $11 \mathrm{~cm}$. It appears that these issues may be interesting enough to warrant further investigation.

In this paper we have investigated in detail only one problem associated with using current GCM sea ice schemes to simulate Snowball ice, but there may be others. One prominent example is that because the dynamics of the viscous flow of ice during a Snowball episode is uncertain (Goodman and Pierrehumbert 2003; Pollard and Kasting 2005; Goodman 2006; Warren and Brandt 2006; Pollard and Kasting 2006), it is unclear what assumptions should be made for salt and brine pocket properties. Ice at the surface in the tropics could be "marine ice" formed by the freezing of seawater and brought to the surface by a "sea ice elevator" driven by surface sublimation (Christie-Blick et al. 1999), or it could be "meteoric ice" formed from compacting snow that flows equatorward from higher latitudes (Goodman and Pierrehumbert 2003). In either case the sea ice schemes used in GCMs, which use salt and brine pocket parameters based on the modern climate, might be inappropriate.

We have neglected solar penetration of sea ice in this paper. The main reason we did this is that not doing so 
resulted in large and unrealistic changes in mean ice surface temperature when we changed the vertical resolution. Additionally, there is a large amount of debate about the appropriate solar penetration parameters for Snowball ice (Warren et al. 2002; Warren and Brandt 2006; Pollard and Kasting 2006). A useful extension of this work would be to carefully consider the effect that solar penetration would have on diurnal cycles in Snowball ice.

In section 4 we focused on the increase in the diurnal cycle that coarse vertical resolution in a sea ice model can produce, and the associated midday melting that can result from this. We did not extensively consider the effects of freezing and melting at the bottom of the sea ice. Bottom melting/freezing during a Snowball is a complicated issue that depends on the details of the ice thickness distribution, viscous flow of ice (Goodman and Pierrehumbert 2003; Pollard and Kasting 2005; Goodman 2006; Warren and Brandt 2006; Pollard and Kasting 2006), the potential of the ocean to concentrate the geothermal heat flux in certain small regions (Adcroft et al. 2001), and the amount of solar radiation that penetrates through the ice and is absorbed by the underlying ocean (Pollard and Kasting 2005; Warren and Brandt 2006; Pollard and Kasting 2006). In any case, it is certain that for a Snowball to deglaciate melting must occur at the surface, and that surface melting is occurring in global climate models that do deglaciate. Therefore, the complicated effects of melting and freezing at the bottom of the ice do not change our conclusion that coarse vertical resolution in the sea ice schemes of GCMs may be leading to false Snowball deglaciations in these GCMs.

Here we have argued that GCM sea ice schemes are overestimating the diurnal cycle in the surface temperature of Snowball ice, which allows the surface temperature to reach freezing unrealistically at midday in some situations. Since these schemes assume that any meltwater is immediately evacuated from the ice surface, a melt-ratchet effect occurs that eventually leads to Snowball deglaciation. Our results therefore show that if the surface temperature diurnal cycle were more accurately simulated, no meltwater would form at midday in many cases and deglaciation would not occur. If the mean surface temperature were sufficiently increased by some climate forcing, however, eventually realistic surface melt would occur. This process would likely be very complicated. Surface meltwater can persist for months even on relatively thin modern sea ice (Fetterer and Untersteiner 1998), so the assumption of immediate evacuation would likely need to be revisited. If meltwater were to remain on the ice surface and refreeze at night, this would require energy and could significantly reduce nighttime cooling. If the meltwater were to remain on the ice surface and not refreeze at night, then melt ponds could absorb significantly more solar radiation than surrounding ice, since they typically have albedos as low as 0.2-0.3 (Langleben 1971). Even if such melt ponds were to refreeze, their albedo would likely stay low because the air bubbles that help to increase ice albedo escape when the ice is melted (Grenfell and Perovich 1984). Investigating the final details of a Snowball deglaciation represents an interesting subject for future research. Such considerations, however, would not alter our conclusions, which concern ice that has not yet reached the freezing point.

\section{Conclusions}

The sea ice schemes used in current global climate models produce inaccurate simulations of the diurnal surface temperature cycle because they do not resolve the penetration depth of the diurnal cycle. This causes unreliable predictions of Snowball deglaciation by global climate models. Increased vertical resolution in global climate model sea ice schemes would improve our understanding of Snowball climate and Snowball termination, and it may also improve the simulation of sea ice in other climates. This increased vertical resolution could be concentrated at the top of the ice so that the associated numerical cost would not be prohibitive.

Acknowledgments. We thank Stephen Warren, Cecilia Bitz, and an anonymous reviewer for comments. DSA was supported by the T. C. Chamberlin Fellowship of the University of Chicago and the Canadian Institute for Advanced Research. IE was supported a Prize Postdoctoral Fellowship through the California Institute of Technology Division of Geological and Planetary Sciences and a NOAA Climate and Global Change Postdoctoral Fellowship administered by the University Corporation for Atmospheric Research. This work was supported by the NSF P2C2 program (ATM-0902844) and NSF ATM-0933936.

\section{APPENDIX}

\section{Solution to the Heat Equation with a Sinusoidal Boundary Condition}

Here we reproduce the long-time solution to the heat equation between two infinite, parallel, horizontally uniform plates with a sinusoidal temperature boundary condition on one of the plates, which was solved by Fourier (1826). This problem is an idealized representation of ice, or any other diffusive medium such as soil, 
forced by a diurnal or seasonal cycle. The full solution to this problem, including transients, is given in Carslaw and Jaeger (1959). The Navier-Stokes equations for a uniform, incompressible, viscous fluid not subject to large-scale pressure gradients and forced by a sinusoidally and transversely oscillating plane boundary simplify to the same equations as those solved here. This is commonly referred to as "Stokes' second problem" and was solved by Stokes (1851). Landau and Lifshitz (1959) and Batchelor (1967) provide more recent and more widely available treatments.

Suppose we are interested in solving for the long-time temperature, $T(z, t)$, in a medium between two parallel plates of infinite extent, where $z$ is the coordinate perpendicular to the plates and $t$ is time. The equation for heat transfer in the medium is

$$
\frac{\partial T}{\partial t}=\kappa \frac{\partial^{2} T}{\partial z^{2}}
$$

where $\kappa$ is the thermal diffusivity. Suppose that at one boundary, $z=H$, we require the boundary condition $T(z=H, t)=T_{b}$, where $H$ is the distance between the two plates and $T_{b}$ is some constant. Suppose that at the other boundary, $z=0$, we require that $T(z=0, t)=$ $T_{m}+T_{v} \cos (\omega t)$, where $\omega$ is the frequency of oscillation and $T_{m}$ and $T_{v}$ are constants. Since $T(z, t)$ is the longtime solution and we are not interested in transients, we do not require an initial condition to solve this problem. Using the ansatz

$$
T(z, t)=T_{m}+\frac{T_{b}-T_{m}}{H} z+T_{v} \Re\left[e^{i(k z-\omega t)}\right],
$$

we find the solution

$$
\begin{aligned}
T(z, t)= & T_{m}+\frac{T_{b}-T_{m}}{H} z \\
& +T_{v} \Re\left\{\frac { 1 } { \beta - 1 } \left[\beta e^{-\left(z / z^{*}\right)} e^{i\left[\left(z / z^{*}\right)-\omega t\right]}\right.\right. \\
& \left.\left.-e^{z / z^{*}} e^{-i\left[\left(z / z^{*}\right)-\omega t\right]}\right]\right\},
\end{aligned}
$$

where $z^{*} \equiv(2 \kappa / \omega)^{1 / 2}=(\kappa P / \pi)^{1 / 2}$, with $P=2 \pi / \omega$ being the period of oscillation, and $\beta \equiv e^{\left(2 H / z^{*}\right)(1-i)}$. For $H \gg z^{*}$, we can approximate Eq. (A3) with

$$
T(z, t) \approx T_{m}+\frac{T_{b}-T_{m}}{H} z+T_{v} e^{-\left(z / z^{*}\right)} \cos \left(\frac{z}{z^{*}}-\omega t\right)
$$

which describes exponentially damped waves of sinusoidal signal propagating into the interior. This approximation is useful for the diurnal cycle $\left(z_{\text {diurnal }}^{*}=0.17 \mathrm{~m}\right)$ in ice as thin as $O(0.5 \mathrm{~m})$ and for the seasonal cycle $\left(z_{\text {seasonal }}^{*}=3.25 \mathrm{~m}\right)$ in glacial ice in the modern climate and thick Snowball sea ice. Equation (A4) makes clear the reason $z^{*}$ is called the $e$-folding depth to which a periodic signal with a given time scale penetrates (section 4 ).

For $z, H \ll z^{*}$, we can approximate Eq. (A3) with

$$
T(z, t) \approx T_{m}+\frac{T_{b}-T_{m}}{H} z+T_{v}\left(1-\frac{z}{H}\right) \cos (\omega t),
$$

In Eq. (A5) the boundary layer extends throughout the entire region so that entire region feels the surface temperature variation. This approximation is useful when considering the seasonal cycle in perennial sea ice in the modern climate (e.g., Eisenman and Wettlaufer 2009), even though $H=O\left(z^{*}\right)$ in this case.

\section{REFERENCES}

Abbot, D. S., and E. Tziperman, 2008: Sea ice, high-latitude convection, and equable climates. Geophys. Res. Lett., 35, L03702, doi:10.1029/2007GL032286.

— and - 2009: Controls on the activation and strength of a high-latitude convective cloud feedback. J. Atmos. Sci., 66, 519-529.

— , and I. Halevy, 2010: Dust aerosol important for snowball deglaciation. J. Climate, 23, 4121-4132.

_ , and R. T. Pierrehumbert, 2010: Mudball: Surface dust and Snowball Earth deglaciation. J. Geophys. Res., 115, D03104, doi:10.1029/2009JD012007.

Adcroft, A., J. R. Scott, and J. Marotzke, 2001: Impact of geothermal heating on the global ocean circulation. Geophys. Res. Lett., 28, 1735-1738.

Bao, H. M., J. R. Lyons, and C. M. Zhou, 2008: Triple oxygen isotope evidence for elevated $\mathrm{CO}_{2}$ levels after a Neoproterozoic glaciation. Nature, 453, 504-506.

_ I. Fairchild, P. Wynn, and C. Spötl, 2009: Stretching the envelope of past surface environments: Neoproterozoic glacial lakes from Svalbard. Science, 323, 119-122.

Batchelor, G., 1967: An Introduction to Fluid Dynamics. 1st ed. Cambridge University Press, 615 pp.

Bitz, C. M., and W. H. Lipscomb, 1999: An energy-conserving thermodynamic model of sea ice. J. Geophys. Res., 104, $15669-15677$.

_ M. Holland, A. Weaver, and M. Eby, 2001: Simulating the ice-thickness distribution in a coupled climate model. J. Geophys. Res., 106 (C2), 2441-2463.

Caldeira, K., and J. F. Kasting, 1992: Susceptibility of the early Earth to irreversible glaciation caused by carbon dioxide clouds. Nature, 359, 226-228.

Carslaw, H., and J. Jaeger, 1959: Conduction of Heat in Solids. 2nd ed. Oxford University Press, $510 \mathrm{pp}$.

Christie-Blick, N., L. E. Sohl, M. J. Kennedy, P. F. Hoffman, and D. P. Schrag, 1999: Considering a Neoproterozoic Snowball Earth. Science, 284, 1087.

Donnadieu, Y., Y. Goddéris, G. Ramstein, A. Nédélec, and J. Meert, 2004: A "Snowball Earth" climate triggered by continental break-up through changes in runoff. Nature, $\mathbf{4 2 8}$, 303-306. 
Eisenman, I., and J. S. Wettlaufer, 2009: Nonlinear threshold behavior during the loss of Arctic sea ice. Proc. Natl. Acad. Sci. USA, 106, 28-32.

Evans, D. A. D., 2000: Stratigraphic, geochronological, and paleomagnetic constraints upon the Neoproterozoic climatic paradox. Amer. J. Sci., 300, 347-433.

Fetterer, E., and N. Untersteiner, 1998: Observations of melt ponds on Arctic sea ice. J. Geophys. Res., 103 (C11), 24 82124835.

Fourier, J., 1826: Théorie du mouvement de la chaleur dans les corps solides. Mémoires de l'Académie Royale des Sciences de l'Institute de France, années 1821 et 1822, Vol. 5, Royal Academy of Science, 153-246.

Goodman, J. C., 2006: Through thick and thin: Marine and meteoric ice in a "Snowball Earth" climate. Geophys. Res. Lett., 33, L16701, doi:10.1029/2006GL026840.

_ rine ice in "Snowball Earth". J. Geophys. Res., 108, 3308, doi:10.1029/2002JC001471.

Grenfell, T., and D. Perovich, 1984: Spectral albedos of sea ice and incident solar irradiance in the southern Beaufort Sea. J. Geophys. Res., 89, 3573-3580.

Hack, J. J., J. E. Truesdale, J. A. Pedretti, and J. C. Petch, cited 2004: SCAM Users' Guide. [Available online at http://www.ccsm. ucar.edu/models/atm-cam/docs/scam/.]

Higgins, J. A., and D. P. Schrag, 2003: Aftermath of a snowball. Geochem. Geophys. Geosyst., 4, 1028, doi:10.1029/2002GC000403.

Hoffman, P. F., and Z.-X. Li, 2009: A palaeogeographic context for Neoproterozoic glaciation. Palaeogeogr. Palaeoclimatol. Palaeoecol., 277, 158-172.

- A. J. Kaufman, G. P. Halverson, and D. P. Schrag, 1998: A Neoproterozoic Snowball Earth. Science, 281, 1342-1346.

Hyde, W. T., T. J. Crowley, S. K. Baum, and W. R. Peltier, 2000: Neoproterozoic 'Snowball Earth' simulations with a coupled climate/ice-sheet model. Nature, 405, 425-429.

Kirschvink, J., 1992: Late Proterozoic low-latitude global glaciation: The snowball Earth. The Proterozoic Biosphere: A Multidisciplinary Study, J. Schopf and C. Klein, Eds., Cambridge University Press, 51-52.

Landau, L., and E. M. Lifshitz, 1959: Fluid Mechanics (in Russian). Pergamon Press, 536 pp.

Langleben, M., 1971: Albedo of melting sea ice in the southern Beaufort Sea. J. Glaciol., 10, 101-104.

Le Hir, G., G. Ramstein, Y. Donnadieu, and R. T. Pierrehumbert, 2007: Investigating plausible mechanisms to trigger a deglaciation from a hard snowball Earth. C. R. Geosci., 339, 274-287.

,,--- , and Y. Goddéris, 2008: Scenario for the evolution of atmospheric $\mathrm{pCO}_{2}$ during a snowball Earth. Geology, 36, $47-50$
Lewis, J. P., A. J. Weaver, and M. Eby, 2006: Deglaciating the snowball Earth: Sensitivity to surface albedo. Geophys. Res. Lett., 33, L23604, doi:10.1029/2006GL027774.

Maykut, G. A., and N. Untersteiner, 1971: Some results from a time-dependent thermodynamic model of sea ice. J. Geophys. Res., 76, 1550-1575.

Pierrehumbert, R. T., 2004: High levels of atmospheric carbon dioxide necessary for the termination of global glaciation. $\mathrm{Na}$ ture, 429, 646-649.

_ 2005: Climate dynamics of a hard snowball Earth. J. Geophys. Res., 110, D01111, doi:10.1029/2004JD005162.

Pollard, D., and J. F. Kasting, 2005: Snowball Earth: A thin-ice solution with flowing sea glaciers. J. Geophys. Res., 110, C07010, doi:10.1029/2004JC002525.

— and - 2006: Reply to comment by Stephen G. Warren and Richard E. Brandt on "Snowball Earth: A thin-ice solution with flowing sea glaciers". J. Geophys. Res., 111, C09017, doi:10.1029/2006JC003488.

Poulsen, C. J., 2003: Absence of a runaway ice-albedo feedback in the Neoproterozoic. Geology, 31, 473-476. , and R. L. Jacob, 2004: Factors that inhibit snowball Earth simulation. Paleoceanography, 19, PA4021, doi:10.1029/ 2004PA001056.

- R. T. Pierrehumbert, and R. L. Jacob, 2001: Impact of ocean dynamics on the simulation of the Neoproterozoic "Snowball Earth." Geophys. Res. Lett., 28, 1575-1578.

Romanova, V., G. Lohmann, and K. Grosfeld, 2006: Effect of land albedo, $\mathrm{CO}_{2}$, orography, and oceanic heat transport on extreme climates. Climate Past, 2, 31-42.

Semtner, A. J., 1976: A model for the thermodynamic growth of sea ice in numerical investigations of climate. J. Phys. Oceanogr., 6, 379-389.

Stefan, J., 1891: Uber die Theorie der Eisbildung, insbesondere uber die Eisbildung im Polarmeere. Ann. Phys., 291, 269-286.

Stokes, G., 1851: On the effect of the internal friction of fluids on the motion of pendulums. Trans. Cambridge Philos. Soc., 9, 8-106.

Trindade, R. I. F., and M. Macouin, 2007: Palaeolatitude of glacial deposits and palaeogeography of Neoproterozoic ice ages. $C$. R. Geosci., 339, 200-211.

Voigt, A., and J. Marotzke, 2010: The transition from the presentday climate to a modern Snowball Earth. Climate Dyn., in press, doi:10.1007/s00382-009-0633-5.

Warren, S. G., and R. E. Brandt, 2006: Comment on "Snowball Earth: A thin-ice solution with flowing sea glaciers" by David Pollard and James F. Kasting. J. Geophys. Res., 111, C09016, doi:10.1029/2005JC003411.

, — - T. C. Grenfell, and C. P. Mckay, 2002: Snowball Earth: Ice thickness on the tropical ocean. J. Geophys. Res., 107, 3167, doi:10.1029/2001JC001123. 